\title{
Mechanical characterisation of the human thoracic descending aorta: experiments and modelling
}

\author{
Claudio M. García-Herrera* ${ }^{a}$. Diego J. Celentano ${ }^{b}$, Marcela A. Cruchaga ${ }^{a}$. Francisco J. Rojo ${ }^{c}$, José Miguel Atienzac, \\ Gustavo V. Guinea" and José M. Goicolea" \\ ${ }^{a}$ Departanento de Ingenieria Mecánica, Universidad de Santiago de Chile. USACH, Avenida Bernardo O'Higgins 3363 , Santiago de \\ Chile, Chile: ${ }^{b}$ Departamento de Ingeniería Mecánica y Metalúrgica, Pontificia Universidad Católica de Chile. Avenida Vicuña \\ Mackenna 4860, Santiago de Chile, Chile: 'E.T.S.I. Caminos, Canales y Puertos, Universidad Politécnica de Madrid. C/Profesor \\ Aranguren s/n, Madrid, Spain
}

\begin{abstract}
This work presents experiments and modelling aimed at characterising the passive mechanical behaviour of the human thoracic descending aorta. To this end, uniaxial tension and pressurisation tests on healthy samples corresponding to newborn, young and adult arteries are performed. Then, the tensile measurements are used to calibrate the material parameters of the Holzapfel constitutive model. This model is found to adequately adjust the material behaviour in a wide deformation range; in particular, it captures the progressive stiffness increase and the anisolropy due to the stretching of the collagen fibres. Finally, the assessment of these material parameters in the modelling of the pressurisation test is addressed. The implication of this study is the possibility to predict the mechanical response of the human thoracic descending aorta under generalised loading states like those that can occur in physiological conditions and/or in medical device applications.
\end{abstract}

Keywords: human aorta; mechanical characterisation; constitutive modelling

\section{Introduction}

The human aorta is an elastic artery whose mechanical properties play a crucial role in damping the pressure wave that occurs inside the vessel and, besides, to influence the blood flow coming from the heart (Nichols and Rourke 1990). Another important aspect closely related to the mechanical behaviour of the artery is the development of surgical treatments and techniques. Some of these treatments (e.g. angioplasty, stent. bypass, aortic valve surgery) involve mechanical features such as the application of loading (usually pressure) and the generation of strain during the repairing or replacement of sick vessels (Kleinstreuer et al. 2008). Moreover, the knowledge of rupture stress and strain in arteries under both normal (physiological) and severe (e.g. automobile accidents or cardiovascular problems) conditions is also a relevant area of current interest (Richens et al. 2002; Field and Richens 2006). These facts justify the need of achieving a betcer understanding of the mechanical response of the artery wall such that the development of constitutive models oriented to this goal can provide useful information for medical diagnosis and therapies of the related pathologies. One of the major challenges involved in the formulation of these models is the definition of realistic and reliable stressstrain relationships of the vessel depending, in turn, on both its type (i.e. ascending, aoric arch, thoracic or abdominal) and age.

Experiments in aortic samples have been carried out in animals (Fung 1993) and. in addition, in human tissues (healthy and with aneurisms) for the ascending (Okamoto et al. 2002: Koullias et al. 2005: García-Herrera 2008), the thoracic (Schulze-Bauer and Holzapfel 2003) and the abdominal (Raghavan et al. 1996, 2006; Martino et al. o1 2006: Vande Geest et al. 2006; Holzapfel et al. 2007: Vorp 2007) aortas. These last three groups have been also mathematically characterised. However, reported in vitro experimental daca and constitutive modelling for healthy human descending aorcas in youngs and newborns are, in particular, racher scarce.

The most common procedure aimed at characterising the passive mechanical behaviour of the human aortic wall is the tensile test. Mohan and Melvin (1982) have used this test to analyse failure properties, anisotropic degree and rate-dependent effects in human descending aoric samples. Lacer, Raghavan et al. (2006) have camied out uniaxial tests in human abdominal aortic aneurisms to assess the tissue degradation as well as the thickness distribution in the aneurism wall.

An alternative approach used to analyse the passive mechanical response of the human aorta is the pressurisation test. In particular, Roy ( 1880 ) assessed. via this test, the nonlinearity of the stress-strain relationship in animal and 
human aortic tissues. Further suldies (Roach and Burton 1957) demonscrated that the aortic stiffness is relatively small for low-pressure levels (i.e. less than those corresponding to the physiological conditions that range from 80 to $120 \mathrm{mmHg}$ ) and very large for high-pressure values. Later, Wolinsky and Glagov (1964) analysed the structural changes experienced by the aortic tissue during this test. concluding that the collagen is mainly involved at high-pressure levels (chis is due to the observed alignment of the collagen fibres at such stress stace), whereas the elastin plays an important role at low-pressure levels. More recently, Acienza et al. (2007) have used this test to snidy the combined effecs of pressure and remperanure on the mechanical response of the human thoracic descending aorta. Experimental measurements obtained with this test have also been employed to fit material parameters of different constitutive models applied to coronary arteries in animals (Fung 1993: Rodríguez et al. 2007).

The aim of this work is to characterise the passive mechanical response of the human thoracic descending aorca. To achieve this purpose, experiments as well as constitutive modelling and determination of material paramecers are perfonmed. The methodology adopted in this work consists of the following steps: (1) fitting of material parameters by means of tensile test data and (2) evaluation of this characterisation in the prediction of the material response in a different stress scate as that present in the pressurisation test. The materials and merhods considered in this suidy are presented in Section 2. In particular, the experimental procedure described in Section 2.1 encompasses two different in vitro tests: uniaxial tension and pressurisation. The tested samples correspond to newborn. young and adult arteries without cardiovascular parhologies. Section 2.2 summarises the Holzapfel constitutive model adopted in this work to describe the material response. The material characterisation via the tensile test is presented in Section 2.3, while Section 2.4 unfolds the main features considered in the analysis of the pressurisation test. The obtained experimental and analytical results included in Section 3 are discussed in Section 4. Specifically, the material characterisation via the tensile tests using the Holzapfel constitutive model is detailed in Section 3. . The procedure to fit the material parameters for such model is particularly analysed. Finally, this material characterisation in the modelling of the pressurisation test is assessed in Section 3.2 where the analytical results are satisfactorily validated with the experimental measurements. The significance of the characterisation of the types of healthy tissues used in this work is twofold: to discuss the effect of the age on the obtained results and to predict their mechanical responses in potential surgical treatments or under extreme cardiovascular conditions (e.g. hypertension).

\section{Materials and methods}

\subsection{Experimental procedure}

\subsubsection{Material}

The human thoracic descending aorta samples tested in this work have been provided by the Hospital Puerta de Hierro at Madrid. All these samples, obtained according to well-established protocols of the ethical committee of such hospital, came from cardiac transplant young and adult donors without previous arterial risk factors (i.e. tissues with low cholesterol levels and absence of arterial pathologies of donors with neither smoking nor diabetes records) whose deachs were not relaced to cardiovascular problems (e.g. automobile accidents. suicide, etc.) and. in addition. from autopsies of newborns that presenced cerebral death. Aorta segments with an approximate length of $50 \mathrm{~mm}$ were considered in this sudy. All the in vitro mechanical tests described in this work have been perfonmed in the same day using samples obtained immediately after their excision (the time between the death of the donors and the moment of excision was around $20 \mathrm{~h}$ in all cases). After harvesting. the aortic tissue specimens were stored in refrigerated saline at $4^{\circ} \mathrm{C}$.

The analysed vessels consisted of thoracic descending aorcas that were classified into three groups, named as A, B and $C$, whose data are, respectively, shown in Tables 1-3. Group A corresponds to newbons, while the respective age intervals for the samples of Groups B and $C$ are 20-36 and 45-60. Although for experimencal purposes the use of more human newbon samples would have been desirable, it should be noted that this kind of arteries are difficult to get due to the low mortality race of newborns and, in addition. the social dramatic conditions involved in such cases.

The composition and structure of the tissues to be mechanically rested were first studied via a histological analysis perfonmed by specialiscs of the Hospical Puerta de Hierro at Madrid. Images of the vessels were taken in order to quantify the fractions of nuclei, elastin and collagen to

Table 1. Data of Group A (newboms).

\begin{tabular}{llcc}
\hline Vessel & Sex & Weight $(\mathrm{kg})$ & Height $(\mathrm{m})$ \\
\hline A1 & F & 2.8 & 0.49 \\
A2 & M & 3.5 & 0.53 \\
\hline
\end{tabular}

Table 2. Data of Group B (youngs).

\begin{tabular}{llccc}
\hline Vessel & Sex & Age (years) & Weight $(\mathrm{kg})$ & Height $(\mathrm{m})$ \\
\hline B1 & F & 32 & 63 & 1.64 \\
B2 & F & 21 & 75 & 1.75 \\
B3 & F & 36 & 49 & 1.65 \\
B4 & F & 20 & 52 & 1.60 \\
B5 & M & 35 & 75 & 1.65 \\
\hline
\end{tabular}


Table 3. Data of Group C (adults).

\begin{tabular}{llccc}
\hline Vessel & Sex & Age (years) & Weight $(\mathrm{kg})$ & Height $(\mathrm{m})$ \\
\hline C1 & F & 60 & 65 & 1.60 \\
C2 & M & 45 & 85 & 1.70 \\
C3 & M & 53 & 73 & 1.66 \\
C4 & M & 57 & 60 & 1.70 \\
C5 & F & 46 & 75 & 1.68 \\
\hline
\end{tabular}

study the distribution of elastin and collagen fibres. This analysis ratified the absence of damaged tissue.

\subsubsection{Tensile test}

The aim of this test is to obtain a stress-strain relationship of the vessel material when it is subjected to a simple (uniaxial) load. This can be properly achieved in nearly straight samples with low curvatures to preclude bending effects (García-Herrera 2008). The strain measure is computed during the test via the displacements of the tensile machine jaws assuming, as observed in the experiments, a nearly homogeneous deformation at the mid-length of the sample. It should be noted that this strain measure was found to be practically the same as that obtained via video-extensometer measurements of the axial displacement between two markers drawn on the sample (García-Herrera 2008); for simplicity, the first procedure was adopted in the present study. This test also provides other important material data as its maximum strength and rupture elongation. Only tests exhibiting rupture at approximately the centre of the sample must be considered. Since the artery wall is composed of three different layers (Fung 1993), valid results are assumed up to the rupture instant of any of such layers.

In this study, the strip samples were obtained from the artery wall. The sample dimensions were selected on the basis of balancing the larger number of specimens with the ease in their manipulation during the experiment (in average, 10 samples were obtained for each available vessel). The chosen sample length and width are plotted in Figure 1, where the thickness was defined by the artery wall whose values ranged in this work from 1.5 to $2.4 \mathrm{~mm}$. The initial width and thickness were measured with an optical extensometer (with precision of $0.001 \mathrm{~mm}$ ). As usual, the samples were cut along the longitudinal and circumferential directions in order to characterise the degree of anisotropy

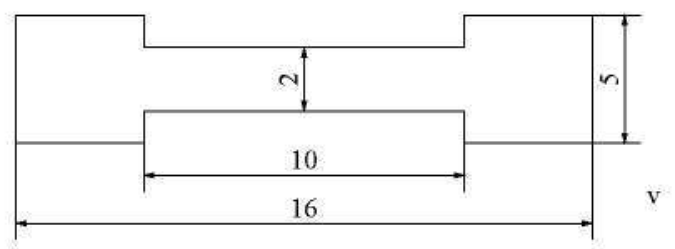

Figure 1. Geometric configuration of the tensile specimen (dimensions in millimetres).

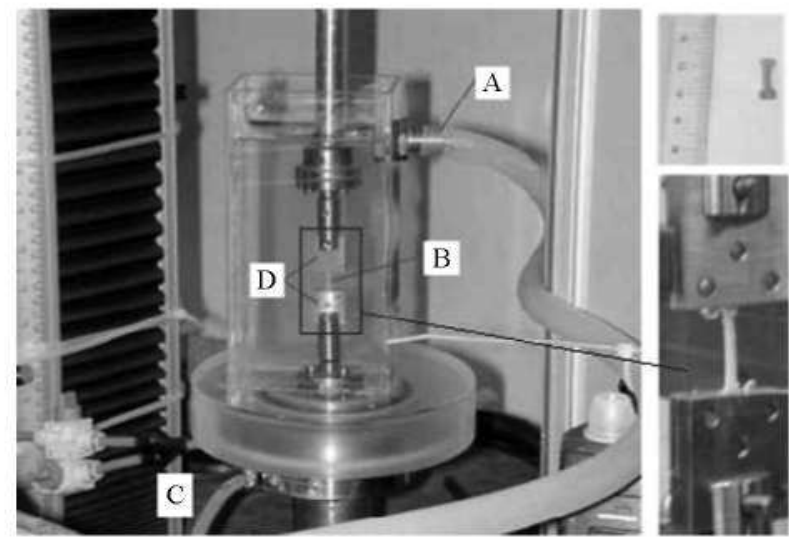

Figure 2. Tensile test assembly. A, physiological serum exit; B, sample; C, physiological serum entry; D, supporting jaws.

in the material response (Mohan and Melvin 1982). These directions are denoted by the angles $0^{\circ}$ and $90^{\circ}$, respectively. Pieces of material belonging to the same artery of those considered for the samples to be tested were stored in formol and sent for histological analysis.

The tests were carried out with the specimens permanently submerged in physiological serum (PBS) at a temperature of $37 \pm 0.5^{\circ} \mathrm{C}$, (see Figure 2). In order to achieve uniform conditions for each sample, a time interval of $10 \mathrm{~min}$ was considered between the end of the assembly and the beginning of the test. To precondition the samples, 10 successive loading cycles were executed up to a stress value of $300 \mathrm{kPa}$. The load cell velocity considered in the tests up to the rupture of the sample was $0.03 \mathrm{~mm} / \mathrm{s}$ (which results in a deformation rate of $15 \% / \mathrm{min}$ approximately). These conditions are assumed to approximately reproduce those corresponding to the physiological behaviour (Mohan and Melvin 1982; Hayashi 1993). In this context, nearly elastic and rate-independent material responses were achieved.

Axial load and axial jaws displacement were recorded during the whole test (with precisions of $0.01 \mathrm{~N}$ and $0.001 \mathrm{~mm}$, respectively; the maximum load cell limit was $10 \mathrm{~N})$. The Cauchy axial stress $\sigma_{1}$ was computed as $F / A$, where $F$ is the axial load and $A$ is current transversal area. The axial stretch $\lambda_{1}$ was calculated as $L / L_{0}$, with $L$ and $L_{0}$ being the current and initial sample lengths, respectively. The current transversal area $A$ is evaluated through the incompressibility condition that leads to $A=A_{0} / \lambda_{1}$, where $A_{0}$ is the initial transversal area of the sample. Moreover, assuming a uniform strain distribution along the sample, the stresses associated with two orthogonal directions perpendicular to the loading direction 1 vanish, i.e. $\sigma_{2}=\sigma_{3}=0$.

\subsubsection{Pressurisation test}

This test is intended to mimic the in vivo loading conditions that exist in the vessel at the interior of the 
human body. In this test, the vessel is subjected to an axial deformation in the tensile machine followed by the application of internal pressure by means of an external compressor (Guinea et al. 2005). To this end, both ends of the specimen are clamped to the jaws. These boundary conditions together with some particular features of the vessel (e.g. relatively low wall thickness-to-diameter and diameter-to-length ratios, material heterogeneity and cylindrical asymmetry) typically cause a complex stress pattern in the sample. However, a nearly uniform stress distribution at least at the central region of the sample may develop when large length-to-diameter and diameter-tothickness ratios are used, e.g. length/diameter $\geq 5$ and diameter/thickness $\geq 10$ (Ogden 1984).

In this study, the following initial ratios were considered: length/diameter $=6.5$ and diameter/thickness $=10$. As mentioned above, these sample dimensions guarantee the development of approximately uniform stress patterns in the wall. The adopted set-up of the pressurisation test was that already described in Guinea et al. (2005). Figure 3 shows the experimental assembly used in this work. The tissue response in both the physiological and high-pressure ranges is specifically analysed.

Internal pressure, axial jaws displacement and external diameter of the vessel were recorded during the whole test. An optical extensometer was used to record the external diameter evolution. Curves of internal pressure $P_{\mathrm{i}}$ vs. circumferential stretch $\lambda_{\theta}$ for different levels of longitudinal stretch $\lambda_{z}$ were obtained. The circumferential stretch was defined as $D / D_{0}$, where $D$ and $D_{0}$ denote the current and initial diameters of the tube, respectively. As in the previous test, the longitudinal stretch was calculated as the ratio Q3 between the current and initial lengths of the tube. Moreover, in order to improve the accuracy of the measurements of $D$, small pieces of tissue located at the outer surface of the sample were removed before being assembled for

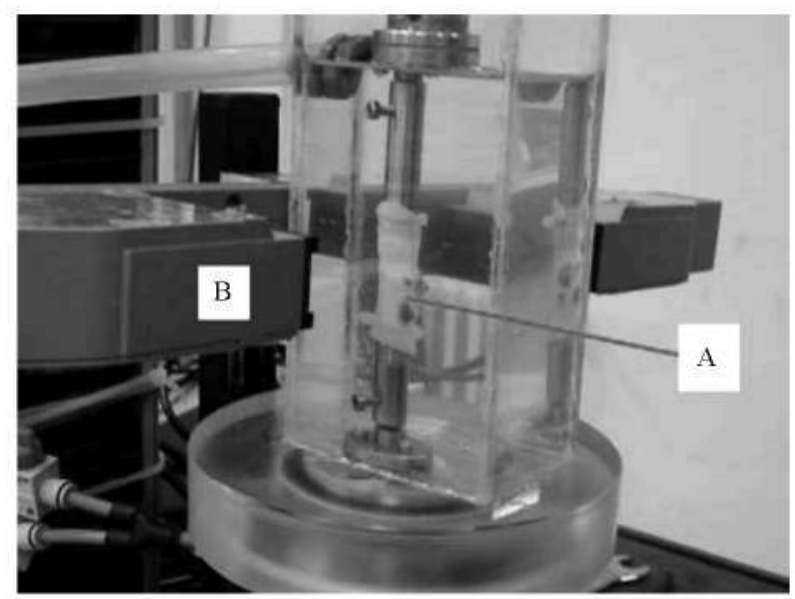

Figure 3. Experimental assembly of the pressurisation test. A, artery; B, optical extensometer. the test. Once again, 10 successive loading cycles up to an axial stretch value of 1.2 followed by a pressure value of $200 \mathrm{mmHg}$ were executed to precondition the samples.

\subsection{Constitutive modelling}

According to the measurements to be presented in Section 3.1 , an elastic and rate-independent material response is considered for the arteries analysed in the present work. Moreover, their behaviours are taken as incompressible due to the large amount of water present in them (Oijen 2003). To this end, hyperelastic constitutive models are used (Ogden 1984; Fung 1993; Holzapfel 2000; Holzapfel et al. 2005; Gasser et al. 2006). In this context, a deformation energy function $W$, assumed to describe the isothermal material behaviour under any loading conditions, can be defined in terms of the right Cauchy deformation tensor $\mathbf{C}=\mathbf{F}^{\mathrm{T}} \cdot \mathbf{F}$, where $\mathbf{F}$ is the deformation gradient tensor and $\mathrm{T}$ is the transpose symbol (note that $\operatorname{det} \mathbf{F}=1$ in this case). Invoking classical arguments of continuum mechanics, the Cauchy stress tensor $\boldsymbol{\sigma}$ is defined as $\boldsymbol{\sigma}=2 \mathbf{F} \cdot(\partial W / \partial \mathbf{C}) \cdot \mathbf{F}^{\mathrm{T}}$.

The Holzapfel model adopted in this work aims at consistently characterising the material anisotropy present in the internal structure of artery tissues (Holzapfel and Gasser 2000). To this end, it assumes that the collagen fibres, which are strongly involved in the mechanical response at moderate-to-high levels of deformation, are helically oriented by means of an approximately constant angle with the artery axis. This model, formulated within the pseudo-invariants framework developed by Spencer (1984), considers the orientations of two symmetric family of fibres defined in the reference (initial) configuration through two unit vectors $\mathbf{a}$ and $\mathbf{a}^{\prime}$, disposed with an angle $\pm \varphi$ with the vessel axis, see Figure 4 (it should be noted that, for simplicity, neither fibre dispersion nor layerspecific responses have been considered, see, e.g. Holzapfel et al. (2005) and Gasser et al. (2006)). The following two invariants can be accordingly defined as $I_{4}=\mathbf{a} \cdot \mathbf{C} \cdot \mathbf{a}$ and $I_{6}=\mathbf{a}^{\prime} \cdot \mathbf{C} \cdot \mathbf{a}^{\prime}$. In this context, the energy

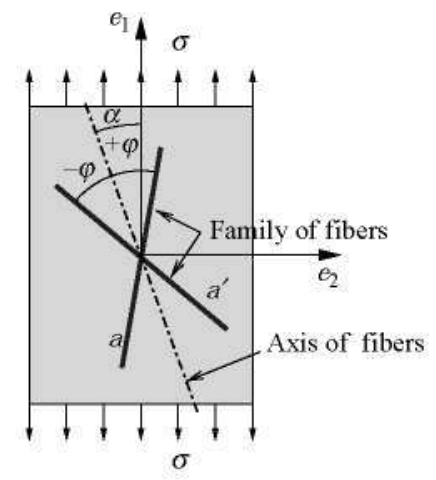

Figure 4. Holzapfel-type material tensile sample. 
function proposed by Holzapfel and Gasser (2000) is written as follows:

$$
W=\frac{\mu}{2}\left(I_{1}-3\right)+\frac{k_{1}}{2 k_{2}} \sum_{i=4,6}\left[\exp \left(k_{2}\left(I_{i}-1\right)^{2}\right)-1\right]
$$

where $I_{1}$ is the first invariant of $\mathbf{C}\left(I_{1}=\operatorname{tr}(\mathbf{C})\right.$. to being the trace symbol) and $\mu, k_{1}, k_{2}, \varphi$ are material parameters (all with positive values). In Equation (1), the first term expressed by a classical neo-Hookean isotropic model is intended to describe the elastin response. while the goal of the other terms is to predict the anisotropic behaviour of the artery given by the directions of the collagen fibres defined, in tum, by the invariants $I_{4}$ and $I_{6}$. Since this model only accounts for macroscopic response, the collagen fraction quantified in the histological analysis is not explicitly considered in this context. Its capabilities in the predictions of the material response in the tensile and pressurisation tests are illustrated in Section 3.

\subsection{Material characterisation via the tensile test}

The aim of this section is to describe the analyticalnumerical procedure aimed at determining the material parameters of the Holzapfel constitutive model briefly presented in Section 2.2 from the uniaxial test measurements to be reported in Section 3.1.

For the Holzapfel constitutive model, it is not possible to obcain a closed expression for the Cauchy stress $\sigma_{1}$ (stress associated with the loading direction 1) and the related strecch $\lambda_{1}$. Therefore, this aspect precludes in this case a straightforward derivation of the related material parameters. According to the procedure described in Ogden (2003), the material response during the tensile test predicted by this model is governed by

$$
\begin{aligned}
\sigma_{1}= & \mu\left(\lambda_{1}^{2}-\frac{1}{\lambda_{1}^{2} \lambda_{2}^{2}}\right)+2 W_{4} \lambda_{1}^{2} \cos ^{2}(\varphi-\alpha) \\
& +2 W_{6} \lambda_{1}^{2} \cos ^{2}(\varphi+\alpha), \\
\sigma_{2}= & \mu\left(\lambda_{2}^{2}-\frac{1}{\lambda_{1}^{2} \lambda_{2}^{2}}\right)+2 W_{4} \lambda_{2}^{2} \sin ^{2}(\varphi-\alpha) \\
& +2 W_{6} \lambda_{2}^{2} \sin ^{2}(\varphi+\alpha)=0,
\end{aligned}
$$

where $W_{i}=\partial W / \partial I_{i}(i=4,6)$ and $\alpha$ denotes the sample orientation (i.e. $\alpha=0^{\circ}$ and $90^{\circ}$ for longitudinal and circumferential samples, respectively, see Figure 4). In this case, the $\sigma_{1}\left(\lambda_{1}\right)$ expression can be obtained by previously solving $\lambda_{2}$ from Equation (3) for an estimated set of material parameters (i.e. $\mu, k_{1}, k_{2}, \varphi$ ) and a given value of $\lambda_{1}$. This last operation is performed through the application of the Newton-Raphson mechod due to the nonlinear nature of Equation (3).
The fitting procedure to detenmine the material parameters for the Holzapfel model is inherently nonlinear. Robust and optimal identification procedures have been developed by Sacks (2000) and Vande Geest et al. (2004, 2006). However, a simpler altemative approach is considered in this work. It basically consists of two steps. respectively, associated with the two elongation zones defined in Section 3.1 (Cacho 2006: Holzapfel 2006):

(1) estimation of parameter $\mu$ through a linear least squares computation aimed at exclusively characterising the isotropic response (i.e. the anisotropic terms of the energy function 1 are neglected at this stage) at low stretching levels and

(2) derivation, at high levels of elongation. of the anisotropic parameters $k_{1}, k_{2}, \varphi$ (with a fixed $\mu$ value) via a nonlinear least squares expression solved with the Levenberg-Marquardt algorithm (Marquardt 1963). In this step, the constraint $\lambda_{2}<1$ is additionally considered in order to obain a more realistic material response.

Finally, the fitting procedure simultaneously considers both the longitudinal and circumferential responses where equal weights are adopted for the two sample directions.

\subsection{Analysis of the pressurisation test}

Although some authors have used this test to derive material parameters for different constitutive models (Schulze-Bauler and Holzapfel 2003; Rodriguez et al. 2007), the aim of the present analysis is to assess the ability of the material characterisation, previously described in Section 2.3 via the tensile tests to properly model the behaviour of the vessel when it is subjected to axial load and internal pressure.

The mechanical response of the pressurised artery is analysed in this work by using the constitutive model presented in Section 2.2. The effects of boch residual stresses and instabilities due to buckling are neglected. It is further assumed here that the artery is a homogeneous thin-walled cylinder with a large length-to-diameter ratio in order to neglect edge effects. At what extent this relatively simple approach is able to realistically describe the average material response during the pressurisation test is anorher objective of this study.

For the loading conditions carried out in the experiments shown in Section 2.1.3, the material responses provided by the Holzapfel constitutive model is given by

$$
\begin{aligned}
P_{\mathrm{i}}= & \frac{2 \mu B_{0}}{D_{0} \lambda_{\theta} \lambda_{z}}\left(\lambda_{\theta}-\frac{1}{\lambda_{\theta}^{3} \lambda_{z}^{2}}\right) \\
& +8 k_{1} \frac{B_{0}}{D_{0} \lambda_{\theta} \lambda_{z}} \exp \left[k_{2}\left(\lambda_{\theta}^{2} \sin ^{2} \varphi+\lambda_{z}^{2} \cos ^{2} \varphi-1\right)^{2}\right] \\
& \times\left(\lambda_{\theta}^{2} \sin ^{2} \varphi+\lambda_{z}^{2} \cos ^{2} \varphi-1\right) \lambda_{\theta} \sin ^{2} \varphi
\end{aligned}
$$


where $B_{0}$ is the initial thickness of the vessel wall. For this test, the average of the initial thickness measurements was $1.8 \mathrm{~mm}$.

\section{Results}

\subsection{Tensile test}

The experimental average stress-stretch curves corresponding to Groups A, B and C for both the longitudinal and circumferential directions are plotted in Figure 5 (the vertical bars denote the standard error. i.e. the ratio between the standard deviation and the square root of the number of specimens).

The stress-stretch curves obtained by applying the least squares fitting described in Section 2.3 to the corresponding experimental data of Groups A, B and C for both the longitudinal and circumferential directions are plotted in Figure 6, whereas Table 4 summarises the derived material parameters for the Holzapfel constitutive model and the normalised mean square root епто $\left(R^{2}\right)$. According to the measurements reported above, the stretch range (1.0-1.8) was chosen for the present material characterisation. since it encompasses a larger deformation interval than that occurring in the pressurisation test presented below. Moreover, the computed $R^{2}$ values show that a good fitting was achieved for all groups.
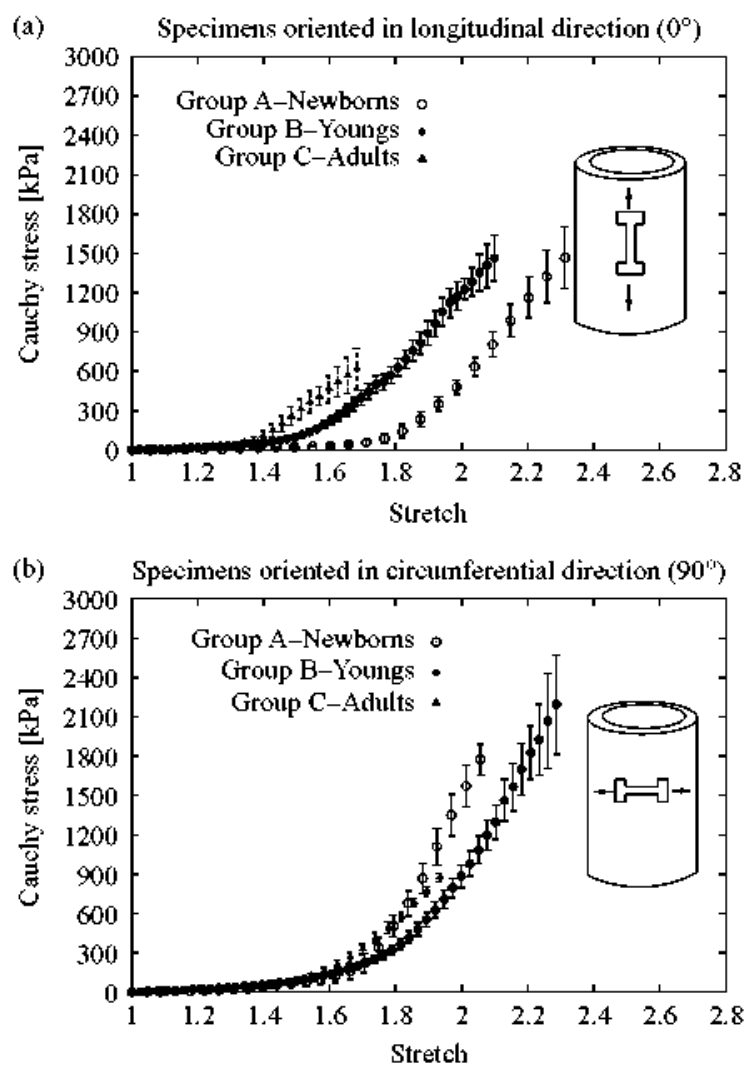

Figure 5. Experimental data of Cauchy stress vs. stretch.
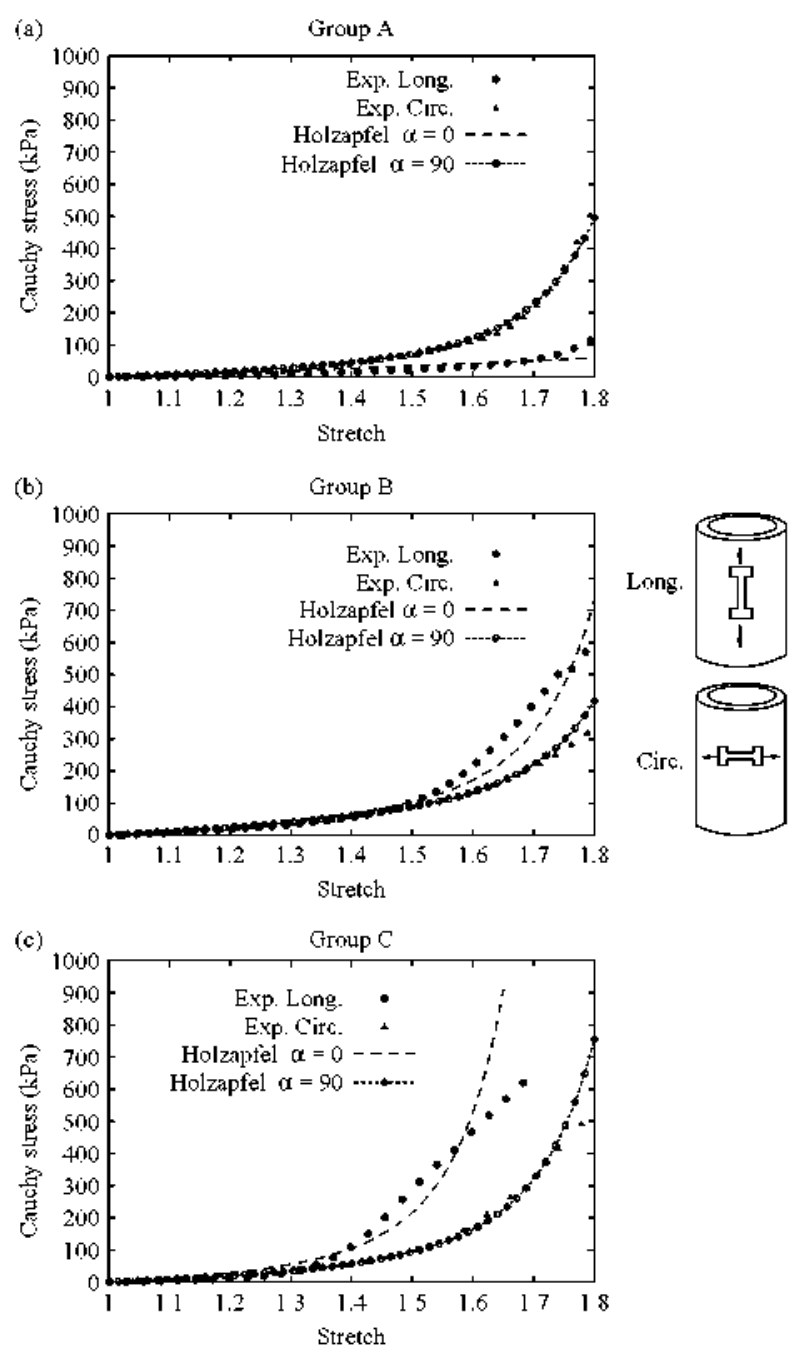

Figure 6. Material characterisation via Cauchy stress vs. stretch curves.

\subsection{Pressurisation test}

Figure 7 shows pressure vs. circumferential stretch experimental data conresponding to Groups $\mathrm{B}$ and $\mathrm{C}$ for two different values of $\lambda_{z}$ (1.0 and 1.2), where the two horizontal lines denote the physiological range of diastole and systole of the cardiac cycle. The value $\lambda_{z}=1.2$ was chosen, since it approximately corresponds to the average stretch measured in the vessel before the excision.

The intenual pressure curve vs. circumferential strench curves obtained with the Holzapfel model (i.e. Equation (4)) is also presented in Figure 7. The material response of

Table 4. Material parameters of the constitutive model.

\begin{tabular}{lcrccc}
\hline Group & $\mu(\mathrm{kPa})$ & $k_{1}(\mathrm{kPa})$ & $k_{2}(\mathrm{kPa})$ & $\varphi\left({ }^{\circ}\right)$ & $R^{2}$ \\
\hline A & 21.972 & 3.011 & 0.7667 & 63.67 & 0.98 \\
B & 37.202 & 14.085 & 3.4752 & 43.88 & 0.95 \\
C & 24.655 & 45.055 & 5.3279 & 42.19 & 0.88 \\
\hline
\end{tabular}



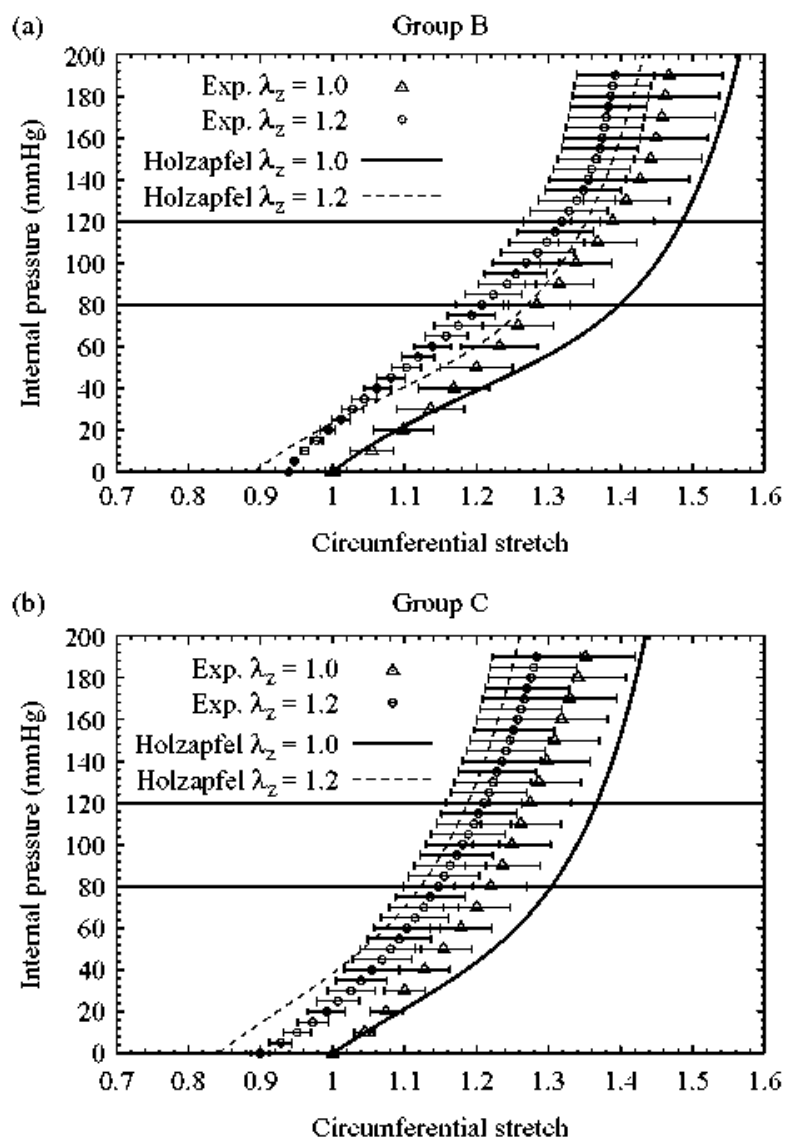

Figure 7. Internal pressure vs. circumferential stretch curves.

Groups B and C is analysed under the two axial stretch conditions described above.

\section{Discussion}

For a given stretch value, the experimental tensile measurements plotted in Figure 5 show that, in general, older vessels present lower rupture defonmations with higher stresses. Minimum rupture stretch values of 1.8 were observed in all cases with the exception of the longitudinal samples of Group $\mathrm{C}$ for which an average rupture stretch of 1.7 was measured. Two zones with different stiffnesses can clearly be identified. At low deformations, the curves show a flexible response with a nearly constant slope. The response of Group A exhibits a wider interval of very low stiffness bounded by $\lambda_{1} \simeq 1.6$. For Groups B and C, the first zone ranges up to $\lambda_{1} \simeq 1.3$ and 1.45 for the longitudinal and circumferential samples, respectively. In this first zone, the material behaviour is clearly isotropic, i.e. its strength is mainly provided by the elastin component of the vessels. For larger deformations. on the orher hand, the slopes of the curves start to increase up to the rupture stage. The material anisotropy in this second elongation zone, reflected in the largest differences between the responses corresponding to both samples directions, is apparent at the very end of the test. This is due to the significant action of the collagen fibres that occurs at high elongation levels.

Although some results for aortas in adults have been found in the literature. it should be noted that they do not strictly correspond to the tissue studied in this work (i.e. in vitro healthy human thoracic descending aortas). For instance, Okamoto et al. (2002) reported an average tensile (uniaxial) nupture elongation of 1.6 for circumferential samples corresponding to dilated human ascending aortas in adults. In this work, the measured final stretch for circumferential samples in adults was 2.0 (see Figure 5(b)).

Figure 6 shows that the Holzapfel model adequately adjusts the overall material response: in particular, the progressive stiffness increases and the anisotropic characters provided by the fibres are properly described. Group A exhibits an average orientation angle $\varphi>45^{\circ}$ (see Table 4) which can be attributed to the low level of longitudinal residual stress present in this very young tissue. Therefore, the circumferential response is more rigid than the longitudinal one at low stretch values. On the other hand, the contrary trend is observed for Groups B and C, i.e. $\varphi<45^{\circ}$, possibly due to the longitudinal residual stress that may act on the aorta during its life (Humphrey 1995). This fact can be also appreciaced in the earlier stiffness increase that occurs in the longitudinal direction samples.

In Figure 7(a), the experimental data corresponding to Group B for $\lambda_{z}=1.0$ exhibit a nearly linear response for pressure values less than $75 \mathrm{mmHg}$. since the elastin is only active in this deformation interval. The effect of the collagen fibres is apparent for pressure values higher than $150 \mathrm{mmHg}$. where the material becomes stiffer. In the longitudinally prestrained case (i.e. $\lambda_{z}=1.2$ ), the axial stress at which the vessel is initially subjected caused a diameter reduction (note that $\lambda_{\theta}<1$ for $P_{\mathrm{i}}=0$ ). However, the average mechanical response of both cases is very similar. Moreover, the sciffness of the vessel also increases in the data corresponding to Group $\mathrm{C}$ plotted in Figure 7(b), but in a more continuous way than that observed for the younger arteries. In general. slightly stiffer responses can be appreciated for the older arteries. In particular, for a pressure value of $P_{\mathrm{i}}=100 \mathrm{mmHg}$, the stretch ranges are 1.27-1.35 and 1.18-1.25 for Groups $B$ and $C$, respectively. Although local buckling occurred for the tests with $\lambda_{z}=1.0$ (due to the compressive load cell force that caused a compressive axial stress in the sample), this undesirable effect practically vanished when a longitudinal prestrain (as the value $\lambda_{z}=1.2$ used in this work which induced a censile resulting load cell force) was applied to the vessel. In addition. it is important to mention that higher dispersion in the diameter measurements was observed for increasing pressure values. These reported experimental data are used below to validate the 
predictions of the Holzapfel model considered in this work.

Moreover, another parameter that is commonly used to characterise the mechanical response of in vivo aortas is the distensibility. Some distensibility values (in $10^{-3} / \mathrm{mmHg}$ ) have been determined for in vino aortas: 2.5 (for an ascending aorta in adults, see Koullias et al. 2005 ) and 3.4 (for only one measurement on an thoracic aorta in an adult, see Schulze-Bauer and Holzapfel 2003). In this work, a value of 2.7 was obtained from the experimental data of Group C shown in Figure 7(b). Other available results are mainly devored to aortas with different types of aneurisms, see e.g. Okamoto et al. (2002), Martino et al. (2006), Raghavan et al. (2006) and Vorp (2007).

The internal pressure curve vs. circumferential stretch curve for the Holzapfel model is also presented in Figure 7 (a) and (b) for Groups B and C. respectively. The differences between the predicted and experimental values for $\lambda_{2}=1.0$ can be attributable to local instabilities that, as mentioned above, occurred during the experiment without longitudinal prestrain. A better agreement. however, is in general achieved for $\lambda_{z}=1.2$. since in this case the buckling effects are practically precluded. Overall, it is seen that the Holzapfel model properly predics the experimental measurements. The stiffness increase occurring at high-pressure levels (i.e. greater than $120 \mathrm{mmHg}$ ) is particularly well described. In this case, the maximum error is less than $8.5 \%$ for the whole test.

\section{Conclusions}

Experiments and modelling focused on the characterisation of the passive mechanical response of the human thoracic descending aorta have been presented. The samples studied in this work encompassed three different groups of healthy arteries: newborns, youngs and adulss. The experimencal data measured in the censile test have been used to determine the material parameters of a Holzapfel constinutive model. In particular, the application of this mechodology to newborn and young tissues is an original contribution of this research. Moreover, the constitutive modelling of the pressurisation test has been experimentally validated. The predictive capabilities and limitations of this model have also been discussed. Overall, the characterisation carried out in this work was found to provide a reasonable and realistic description of the mechanical behaviour of the human thoracic descending aorta under different loading conditions. The limitations of this work that have to be addressed in further research are mainly related to two different aspects: assumptions in the constitutive modelling (e.g. the consideration of layer-based laws, rate-dependent effects and damage evolution has to be explored) and use of simplified analytical solutions in problems with complex stress and strain patterns (e.g. the opening ring test).

\section{Acknowledgements}

The authors wish to express their appreciation to Dr R. Burgos. Dr C. García-Montero, Dr J. Goicolea of the Hospital de Puerta de Hierro at Madrid for the provision of arterial tissues analysed in this work. The supports provided by the Ministerio de Ciencia e Innovación (Spain) through the project MAT2009-10258, by the Comunidad de Madrid (Spain) through the programme MADR.IB-CM/S-SAL/0312/2006, the Fundación Marcelino Botin, the post-doctoral programme at the Universidad de Santiago de Chile (USACH) and the FONDECYT Project No. 11090266 of the Chilean Council of Research and Technology (CONICYT) are all gratefully acknowledged.

\section{References}

Atienza JM, Guinea GV. Rojo FJ, Burgos RJ, García-Montero C. Goicolea FJ, Argoncillo P. Elices M. 2007. The influence of pressure and temperature on the behavior of the human aorta and carotid arteries. Rev Esp Cardjol, 60:259-267.

Cacho F. 2006. Constitutive models for soft biological tissues [PhD thesis]. [in Spanish]: Universidad de Zaragoza.

Field M, Richens D. 2006. Anticipatory valsalva-type response as a contributory factor in low impact blunt traumatic aortic rupture. Med Hypotheses. 67:87-92.

Fung Y. 1993. Biomechanics: mechanical properties of living tissues. New York: Springer-Verlag.

García-Herrera CM. 2008. Mechanical hehaviour of the human ascending aorta: characterization and numerical simulation [PhD thesis]. [in Spanish]: Universidad Politécnica de Madrid.

Gasser CT, Ogden RW, Holzapfel GA. 2006. Hyperelastic modelling of arterial layers with distributed collagen fibre orientations. J R Soc Interface. 3:15-35.

Guinea GV, Atienza JM. Elices M, Argoncillo P. Hayashi K. 2005. Thermomechanical behavior of human carotid arteries in the passive state. AJP - Heart Circ Physiol. 288:2940-2945.

Hayashi K. 1993. Experimental approaches on measuring the mechanical properties and constitutive laws of arterial walls. J Biomech Eng. 115:481-488.

Holzapfel G. 2000. Non linear solid mechanics. Chichester: Wiley.

Holzapfel G. 2006. Determination of material models for arterial walls from uniaxial extension tests and histological structure. J Theor Biol. 238:290-302.

Holzapfel GA, Gasser TC. 2000. A new constitutive framework for arterial wall mechanics and a comparative study of material models. J Elast. 61:1-48.

Holzapfel GA, Sommer G, Auer M, Regitnig P, Ogden RW. 2007. Layer-specific 3D residual deformations of human aortas with non-atherosclerotic intimal thickening. Ann Biomed Eng. 35:530-545.

Holzapfel GA, Sommer G, Gasser CT, Regitnig P. 2005. Determination of layer-specific mechanical properties of human coronary arteries with nonatherosclerotic intimal thickening and related constitutive modeling. Am J Physiol Heart Circ Physiol. 289:H2048-H2058.

Humphrey J. 1995. Mechanics of the arterial wall: review and directions. Crit Rev Biomed Eng. 23:1-162.

Kleinstreuer C, Li Z. Basciano C. Seelecke S. Farber M. 2008. Computational mechanics of nitinol stent grafts. J Biomech. $41: 2370-2378$.

Koullias G, Modak R, Tranquilli M. 2005. Mechanical deterioration underlies malignant behavior of aneurysmal human ascending aorta. J Thorac Cardjovase Surg. 130:677e1-677e9. 
Marquardt D. 1963. An algorithm for least squares. SIAM J Appl Math. 11:431-441.

Martino ESD, Bohra A. Geest JPV. Gupta N, Makaroum MS, Vorp DA. 2006. Biomechanical properties of nuptured vs. electively repaired abdominal aortic aneurysm wall tissue. $\mathrm{J}$ Vasc Surg. 43(3):570-576.

Mohan D, Melvin J. 1982. Failure properties of passive human aortic tissue. I uniaxial tension test. $\mathrm{J}$ Biomech. 15(11):887-902.

Nichols W, Rourke MO. 1990. McDonald's flow in arteries: theoretical, experimental and clinical principles. 3rd ed. New York: Oxford University Press.

Ogden RW. 1984. Non-linear elastic deformations. New York: Dover Publications, Inc.

Ogden RW. 2003. Nonlinear elasticity with application to material modelling. Warsaw: Polish Academy of Sciences.

Oijen CV. 2003. Mechanics and design of liber-reinforced vascular prostheses [ $\mathrm{PhD}$ thesis]. Technische Universiteit Eindhoiven.

Okamoto RJ, Wagenseil JE, DeLong WR, Peterson SJ, Kouchoukos NT, Sundt TM, III. 2002. Mechanical properties of dilated human ascending aorta. Ann Biomed Eng. 30:624-635.

Rachev A. Greenwald S. 2003. Residual strain in conduit arteries. J Biomech. 36:661-670.

Raghavan ML, Kratzberg J, de Tolosa EMC, Hanaoka MM, Walker P. da Silva ES. 2006. Regional distribution of wall thickness and failure properties of human abdlominal aortic aneurysm. J Biomech. 39:3010-3016.

Raghavan ML, Webster M, Vorp DA, 1996, Ex vivo biomechanical behavior of abdominal aortic aneurysm assessment using a new mathematical model. Ann Biomed Eng. 24(5):573-582.

Richens D, Field M. Neale M. Oakley C. 2002. The mechanism of injury in blunt traumatic rupture of the aorta. Eur $\mathrm{J}$ Cardiothorac Surg. 21:288-293.

Roach MR, Burton AC. 1957. The reason for the shape of the distensibility curves of arteries. Can J Biochem Physiol. 35:681-690.

Rodríguez J, Goicolea JM, Gabaldón F. 2007. A volumetric model for growth of arterial walls with arbitrary geometry and loads. J Biomech. 40:961-971.

Roy CS. 1880. The elastic properties of the arterial wall. J Physiol. 3:125-162.

Sacks MS. 2000. Biaxial mechanical evaluation of planar biological materials. J Elast. 61:199-246.

Schulze-Bauer C, Holzapfel G. 2003. Determination of constitutive equations for human arteries from clinical data. J Biomech. 36:165-169.

Spencer A. 1984. Continuum theory of the mechanics of fibrereinforced composites. CISM. 282:1-32.

Vande Geest JP, Di Martino ES. Vorp DA. 2004. An analysis of the complete strain field within Flexcercell ${ }^{\mathrm{TM}}$ membranes. J Biomech. 37:1923-1928.

Vande Geest JP, Sacks MS, Vorp DA. 2006. The effects of aneurysm on the biaxial mechanical behavior of human abdominal aorta. J Biomech. 39:1324-1334.

Vorp DA. 2007. Biomechanics of abdominal aortic aneurysm. J Biomech. 40:1887-1902.

Wolinsky H. Glagov S. 1964. Stnictural basis for the static mechanical properties of the aortic media. Circ Res. $14: 400-413$. 